\title{
Dependencia de la tensión superficial con el método de medida
}

\author{
Julio Alberto Clavijo Penagos ${ }^{12 \star}$ \\ ${ }^{1}$ Grupo de Materiales Fotoactivos, Departamento de Química, Universidad de \\ América, Bogotá, Colombia \\ 2 Laboratorio de investigaciones básicas, Facultad de Ciencias, Universidad Nacional \\ de Colombia, Bogotá, Colombia
}

FeCha de Entrega: Enero 26 DE 2012

FeCha de EVAluación: FeBrero 16 DE 2012

FECHA DE APROBACión: MAYO 3 DE 2012

\begin{abstract}
Resumen Este trabajo presenta resultados experimentales que ilustran de manera clara y evidente la gran dependencia que tiene la tensión superficial con respecto al método de medida que se usa para determinarla. Se presentan resultados de tensión superficial de soluciones acuosas del aminal macrocíclico 1,3,5,7-Tetraazatriciclo[3.3.1.1 $\left.{ }^{3,7}\right]$ decano, conocido como HMT, obtenidos a $25,0{ }^{\circ} \mathrm{C}$ mediante dos métodos estáticos distintos, el método del anillo de DuNuoy y el método del volumen de gota. Se discute acerca de la variación que presenta la propiedad con respecto a las características propias de los métodos usados para determinarla y la importancia de dar soporte a la escogencia de un método de determinación experimental de la tensión superficial sobre un adecuado conocimiento de las propiedades fisicoquímicas e incluso termodinámicas del sistema acuoso a estudiar.
\end{abstract}

Palabras Clave: tensión superficial, HMT, anillo de DuNuoy, volumen de gota, propiedades superficiales.

Abstract This article presents experimental results that clearly illus-
trate the big dependence superficial tension has with respect to the
measure method used to determine it. There were superficial tension
results of watery solutions belonging to macrocyclic aminal $1,3,5,7-$
Tetraazatricycle[3.3.1. $1^{3,7}$ ]ecane, known as HMT, obtained at $25,0{ }^{\circ} \mathrm{C}$
through two different static methods: the Du Noüy Ring Method and
the Drop Volume Method. We discussed about the variation the property
presents with respect to the characteristics belonging to the methods
used to determine it and to the importance of focusing the selection of an
experimental determining method of superficial tension on an adequate
knowledge of the physicochemical and thermodynamic properties of the
watery system to be studied.

Keywords: superficial tension, HMT, DuNoüy Ring, Drop Volume, superficial properties.

^ Químico UN, Doctor en Cienacias - Química UN. Áreas de interés: fisicoquímica y termodinámica de soluciones acuosas termodinámica de superficies acuosas, astronomía, cosmología, astrofísica. jaclavijop@gmail.com 


\section{Introducción}

De todas las propiedades fisicoquímicas y termodinámicas que usualmente se estudian con respecto a las soluciones acuosas, ya sea en el ámbito industrial, biológico o académico, la tensión superficial es una de las de mayor aplicación e importancia, sobre todo en la ingeniería alrededor de la fabricación de una muy extensa variedad de productos químicos, farmacéuticos y alimenticios, entre muchos otros campos de aplicación. Sin embargo, la tensión superficial no es una propiedad tan simple como puede parecer, en especial, en cuanto a la forma experimental de determinarla, empezando porque no es una propiedad presente en toda la extensión de una solución acuosa, como la densidad o la temperatura, sino presente solamente en la región de la solución en contacto con otra fase distinta, sea otra solución distinta o el aire. Por ello, no es difícil encontrar en la literatura una gran cantidad de casos en los cuales los valores que diferentes investigadores reportan para la tensión superficial de un mismo sistema estudiado, a una misma temperatura y bajo los mismos conceptos teóricos de análisis, resultan divergentes y a veces hasta inaceptablemente lejanos, siendo experimentadores reconocidos.

En este orden de ideas, el objetivo de este trabajo es presentar resultados experimentales de investigación que claramente demuestran la importancia de conocer adecuadamente el sistema al cual se le desea determinar sus propiedades superficiales, para así escoger el método experimental más conveniente para obtener resultados representativos y confiables. Se presenta el caso de las soluciones acuosas del aminal macrocíclico 1,3,5,7-Tetraazatriciclo[3.3.1.1 $\left.1^{3,7}\right]$ decano, conocido como HMT, por ser una molécula de altísima solubilidad [6] y comportamiento superficial no común $[8,11]$, y por tanto, poco reportada en la literatura.

\subsection{Fundamentos teóricos de la tensión superficial}

Si bien el objetivo de este trabajo no es presentar en extenso la teoría alrededor de la tensión superficial, es necesario tener un conocimiento básico de la misma para entender la influencia del método de determinación sobre los valores experimentales según el sistema estudiado; un tratamiento detallado sobre la teoría y la experimentación alrededor de la tensión superficial se encuentra fácilmente en la literatura [1,2]. Brevemente, si el área de la superficie presente entre dos fases puras 1 y 2 se denomina $A$, existe un trabajo reversible necesario para aumentar el área interfacial que está dado por el producto $\sigma^{1,2} d A$. La magnitud $\sigma^{1,2}$ se denomina tensión interfacial (cuando las dos fases son líquidas) o tensión superficial (cuando una de las fases es un gas - aire, por ejemplo) [1]. Como el trabajo para aumentar un área superficial o interfacial siempre es positivo, el valor de la tensión superficial siempre es positivo. De esta forma, se puede extender el concepto para hablar de tensión superficial para líquidos puros en contacto (equilibrio) con su propio vapor y de soluciones en equilibrio con el vapor del solvente, si el único componente volátil es el solvente, o con el vapor formado por todos los componentes volátiles de la solución, caso común en mezclas acuosas de líquidos. En el caso práctico más común, el solvente es agua y el soluto es un componente no volátil que afecta la tensión superficial del solvente. De esta 
forma, una solución acuosa tiene una sola interfase solución-aire, por lo que la tensión superficial de la solución suele denominarse simplemente como $\sigma$.

La tensión superficial tiene unidades de energía o trabajo por unidad de área. Las unidades SI para $\sigma$ son $\mathrm{J} / \mathrm{m}^{2}=\mathrm{N} / \mathrm{m}$. Como los valores experimentales de $\sigma$ suelen ser del orden de $10^{-3} \mathrm{~N} / \mathrm{m}$, también es frecuente encontrar valores de $\sigma$ expresados como $\mathrm{mN} / \mathrm{m}$. Normalmente, $\sigma$ disminuye con el aumento de la temperatura hasta el punto crítico del solvente.

Cuando un soluto está presente al interior del agua, el establecimiento de interacciones soluto-soluto y soluto-solvente da lugar a la modificación estructural del solvente, lo cual genera que haya una distribución del soluto al interior del solvente pero también sobre la interfase aire-solución [2]. Si las interacciones soluto-solvente son mucho mayores en comparación a las interacciones solutosoluto, frecuentemente se observa que la solubilidad del soluto en el solvente es alta, pero puede pasar que si el soluto tiene en su estructura partes apolares y polares, las partes polares se asocian preferentemente con las moléculas de agua muy bien pero no así las partes apolares del soluto. Entonces, el agua se estructura de manera que una gran cantidad de moléculas de soluto quedan bien disueltas (asociadas) al interior de la solución pero también ocurre una migración de moléculas de soluto hacia la interfase (adsorción), quedado las partes polares del mismo asociadas a las moléculas de solvente presentes en la región superficial y las partes apolares orientadas hacia la fase apolar o hacia el aire [3]. Sin embargo, llega un momento cuando la superficie ya ha alojado la mayor cantidad posible de soluto y como no puede permitir mayor ocupación, las moléculas de soluto que quieren migrar a la superficie no podrán hacerlo y optan por autoasociarse formando grupos al interior de la solución acuosa, grupos donde las partes apolares del soluto se asocian unas con otras protegidas del contacto con el agua por las partes polares del mismo que sí permanecen en contacto con el agua, fenómeno llamado agregación micelar, por lo que dichos grupos de solutos se denominan micelas $[3,4]$.

Todo esto indica que un soluto que migre hacia la superficie de una solución inevitablemente altera la tensión superficial del solvente, y un soluto que así se comporte se denomina agente superficial o surfactante. A mayor porción apolar presente en la estructura del soluto, mayor alteración de la tensión superficial del solvente. En términos ideales, el valor de $\sigma$ debe ser el mismo no importando el método escogido para determinarlo, ya sea para un líquido puro o para una solución dada. Sin embargo, la realidad es que el valor de $\sigma$ que se encuentra para un sistema dado depende grandemente del método escogido para determinarlo cuando el sistema estudiado no se comporta de manera común. En general, cuando el surfactante tiene grandes partes apolares, se acumula rápidamente sobre la superficie, por lo que afecta grandemente la tensión superficial del solvente aún incluso a concentraciones del orden de las ppm [3,5], como es el caso de los detergentes comunes. En estos casos comunes, el método de determinación de la tensión superficial no afecta de manera apreciable los valores de $\sigma$ obtenidos para la solución porque el efecto del surfactante ya altera de manera extensa y suficiente la estructura del solvente en la superficie, por lo que un método 
invasivo de determinación de $\sigma$ (como los métodos de placa o de anillo) no va a alterar aún más la superficie. Pero en ciertos casos, el soluto no provoca un efecto surfactante grande sino muy escaso pero suficiente para alterar la tensión superficial del solvente, pero como la estructura del solvente está menos alterada de lo común, el método usado para la determinación de $\sigma$ puede llegar a perturbar inconvenientemente la superficie y ser así responsable de las variaciones entre los valores de la propiedad encontrados mediante distintas técnicas, como a continuación los resultados presentados en este trabajo lo demuestran. El lector puede remitirse a la literatura para una mayor comprensión de las técnicas experimentales de determinación de la tensión superficial [2].

\section{Parte experimental}

Se determinó la tensión superficial de diversas soluciones acuosas de HMT a 25, 0 ${ }^{\circ} \mathrm{C}$ por medio de un método superficialmente invasivo (anillo de DuNuoy) y de dos métodos no invasivos (volumen de gota y gota pendiente) para determinar si el método escogido afecta los resultados de tensión superficial de las soluciones. El soluto HMT fue adquirido comercialmente (Sigma-aldrich), con pureza $\geq$ 99, $0 \%$. El agua utilizada para la preparación de las soluciones presentó un valor de tensión superficial a $25,0{ }^{\circ} \mathrm{C}$ de $71,44 \mathrm{mN} / \mathrm{m} \pm 0,09 \mathrm{mN} / \mathrm{m}$ (anillo) y de $71,42 \mathrm{mN} / \mathrm{m} \pm 0,083 \mathrm{mN} / \mathrm{m}$ (volumen de gota). En el caso de las soluciones trabajadas mediante el método del anillo, se realizaron siete soluciones cubriendo todo el intervalo de solubilidad, es decir, hasta 43, $32 \%$ p/p [6]; en el caso del método de volumen de gota, se prepararon nueve soluciones. Todas las soluciones se prepararon por pesada. Las determinaciones realizadas mediante el método del anillo se hicieron usando un tensiómetro Tantec ST-Plus, con precisión de 0,1 $\mathrm{mN} / \mathrm{m}$; Las determinaciones realizadas mediante el método del volumen de gota se hicieron usando un tensiómetro Lauda TVT2, con precisión de 0,01 mN/m.

\section{Resultados y discusión}

\subsection{Tensión superficial de soluciones acuosas de HMT}

En el cuadro 1 se presentan los resultados experimentales de tensión superficial de las soluciones acuosas de HMT a $25,0{ }^{\circ} \mathrm{C}$ obtenidos a través del método del anillo de DuNuoy, y en cuadro 2 se presentan los resultados experimentales de tensión superficial de las soluciones acuosas de HMT a $25,0{ }^{\circ} \mathrm{C}$ obtenidos a través del método del volumen de gota.

Comparando estos resultados del cuadro 1 y de la figura 1 con lo encontrado en la literatura [7], se encuentra que al utilizar la técnica del volumen de gota, los valores son comparables y consistentes en la tendencia que se observa en ellos, pero no así con los valores obtenidos mediante el método del anillo de DuNuoy, los cuales se alejan bastante de los obtenidos por volumen de gota, aún a muy bajas concentraciones. Esto puede deberse a la naturaleza propia tanto del sistema acuoso como del método utilizado para la determinación de la tensión superficial. 
La alta solubilidad del HMT en agua a $25,0{ }^{\circ} \mathrm{C}[6]$ indica que las interacciones soluto-solvente son dominantes en las mismas, lo cual se explica en virtud de los cuatro átomos de nitrógeno que cada molécula de HMT posee, tal como recientes reportes lo demuestran incluso a temperaturas alrededor de la temperatura de máxima densidad del agua, donde la estructura del agua es especialmente sensible a las interacciones soluto-soluto y soluto-solvente [8,9,10,11,12]. Así, esta misma presencia de los cuatro nitrógenos por molécula de HMT hace que las moléculas de agua puedan estructurarse alrededor de las mismas de manera que muchas de ellas pueden asociarse con el agua a través de enlaces de hidrógeno. Sin embargo, como la molécula de HMT es globular con partes apolares (los grupos metileno), es muy posible algunas de ellas migren hacia la superficie producto de la hidratación hidrofóbica, llegando así a la superficie y alterando la tensión superficial; este efecto es natural que sea más evidente al aumentar la concentración, pues el agua cada vez es menos capaz de solubilizar al soluto en tanto aumente la concentración del mismo, tal como se nota en la figura 1.

\begin{tabular}{cl}
\hline$X$ HMT $\sigma \mathrm{mN} / \mathrm{m}$ \\
\hline 0,0921 & $56,54(0,42)$ \\
\hline 0,0892 & $57,96(0,52)$ \\
\hline 0,0772 & $61,66(0,49)$ \\
\hline 0,0511 & $67,76(0,18)$ \\
\hline 0,028 & $69,58(0,08)$ \\
\hline 0,013 & $70,96(0,13)$ \\
\hline 0 & $71,44(0,09)$ \\
\hline
\end{tabular}

Cuadro 1. Tensiones superficiales de soluciones acuosas de HMT, a $25,0{ }^{\circ} \mathrm{C}$ por el método del Anillo de DuNuoy. Los números entre paréntesis indican la desviación estándar del valor reportado.

En este orden de ideas, dado que el método del anillo de DuNuoy requiere determinar la fuerza necesaria para lograr la ruptura de la superficie para hallar el valor de la tensión superficial, se puede afirmar que este método resulta poco adecuado para determinar la tensión superficial de soluciones de solutos de este tipo, siendo así mejores aquellos métodos donde no se requiera romper la superficie, y por tanto, los valores de tensión superficial sean realmente representativos de la superficie del sistema acuoso analizado.

A partir de los resultados reportados en este trabajo, se puede afirmar, tal como se pude encontrar en la literatura $[8,11]$ que la adsorción del HMT en la superficie líquido vapor de las soluciones es aparentemente muy débil, por lo que el equilibrio se ve alterado fácilmente por el método de medida. Entonces, como el método del anillo es un método superficialmente invasivo, es decir, que altera la región superficial, la adsorción se ve alterada y es necesario alcanzar un nuevo 
equilibrio, pero como de por sí la adsorción es muy débil, el establecimiento de un nuevo equilibrio requiere bastante tiempo. Todo lo anterior hace muy complicado obtener valores reproducibles de la tensión superficial de las soluciones acuosas de HMT mediante un método superficialmente invasivo. En este orden de ideas, los resultados que se obtienen por métodos que alteran la superficie no son comparables con aquellos resultados obtenidos por métodos no intrusivos, como los métodos del volumen de gota, del peso de gota o de gota pendiente.

\begin{tabular}{cc}
\hline$X$ HMT & $\sigma \mathrm{mN} / \mathrm{m}$ \\
\hline 0 & $71,42(0,08)$ \\
\hline 0,02107 & $71,35(0,01)$ \\
\hline 0,03079 & $71,26(0,02)$ \\
\hline 0,04008 & $71,09(0,02)$ \\
\hline 0,04929 & $70,84(0,03)$ \\
\hline 0,05985 & $70,36(0,09)$ \\
\hline 0,06944 & $69,88(0,26)$ \\
\hline 0,08106 & $69,07(0,05)$ \\
\hline 0,09161 & $68,26(0,06)$ \\
\hline 0,09322 & $68,12(0,05)$ \\
\hline
\end{tabular}

Cuadro 2. Presión superficial de soluciones acuosas de HMT a $25,0{ }^{\circ} \mathrm{C}$ por el método del volumen de gota. Los números entre paréntesis indican la desviación estándar del valor reportado.

En la figura 1 se hace la comparación de los resultados obtenidos mediante los dos métodos escogidos en este trabajo para discutir sobre el posible efecto del método experimental sobre la tensión superficial. Al observar la figura 1 se nota claramente que la adsorción del HMT en la superficie líquido-vapor de la solución acuosa es muy diferente al comportamiento usual de un surfactante común, puesto que la disminución de $\sigma$ en la soluciones acuosas de HMT es bastante pequeña en comparación a soluciones acuosas de dodecilsulfato de sodio (SDS) (figura 2). Esto sustenta la idea de que las moléculas de HMT se adsorben de manera muy débil, lo cual explica lo observado en los resultados de este estudio, porque si la fuerza de la adsorción del HMT es muy débil, una alteración del equilibrio hace que el mismo se reestablezca muy difícilmente, por lo que la determinación de la tensión superficial de las soluciones acuosas de HMT a través de métodos intrusivos, como el del anillo de DuNuoy, es poco reproducible, tal como los resultados de este trabajo lo muestran.

Agradecimientos. J. A. Clavijo agradece al Señor Jesús por la oportunidad de desarrollar este trabajo en el laboratorio de Superficies de la UNAM, así como al Dr. Jesús Gracia Fadrique, director del laboratorio. 


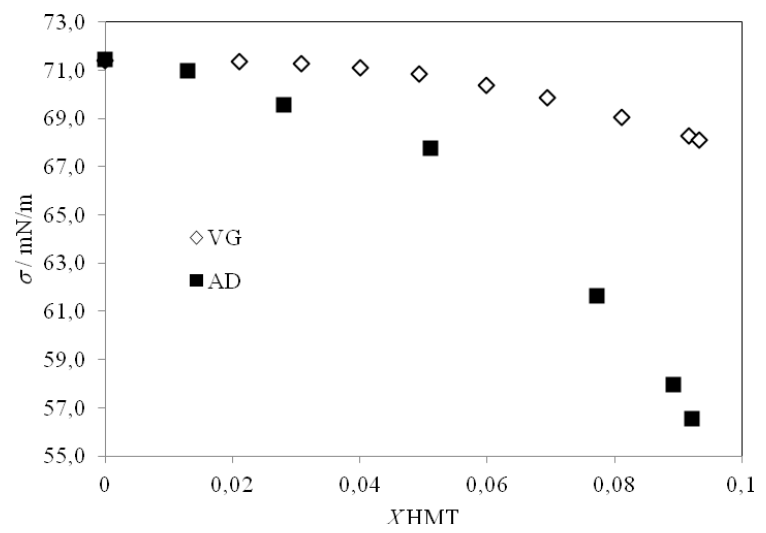

Figura 1. Tensión superficial de soluciones acuosas de HMT a $25.0{ }^{\circ} \mathrm{C}$ por el método del anillo de DuNuoy (AD) y por el método de volumen de gota (VG).

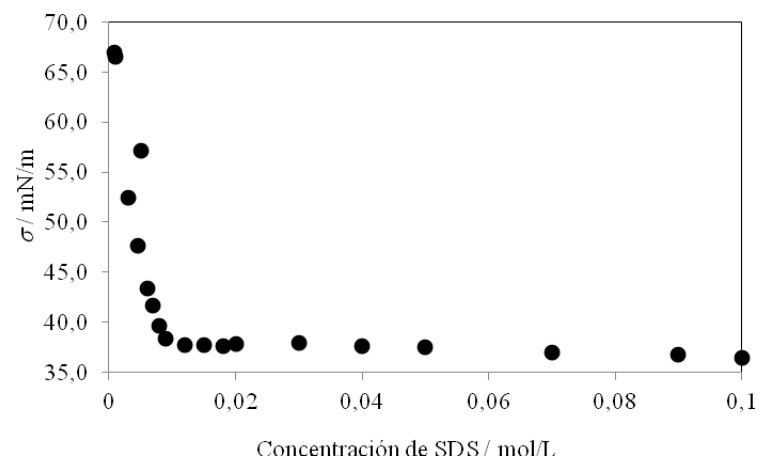

Figura 2. Tensión superficial de soluciones acuosas de SDS a $25.0^{\circ} \mathrm{C}$. Valores tomados de la referencia [5].

\section{Referencias}

1. Levine, I.N.: Fisicoquímica Vol. 1, 5 ed. Madrid: McGraw-Hill/Interamericana de España. (2004)

2. Adamson, A.W., Gast A.P.: Physical chemistry of surfaces, New York: John Wiley \& Sons, Inc. (1997)

3. Viades-Trejo, J., Amigo, A., Gracia-Fadrique, J.: Fluid Phase Equilib. 250, 158-164. (2006)

4. Novelo-Torres, A.M., Gracia-Fadrique, J.: Idealidad en superficies fluidas, Educ. quím., 14[4], p. 220-224, (2003)

5. Castro, M.J.L., Ritacco, H., Kovensky, J., Fernández-Cirelli, A.J.: Chem. Educ. 78, 347-348. (2001) 
6. Blanco, L.H., Sanabria, N.R., Dávila M.T.: Solubility of 1,3,5,7-tetraazatricyclo[3.3.1.1 $1^{3,7}$ decane (HMT) in water from $275.15 \mathrm{~K}$ to $313.15 \mathrm{~K}$. Thermochim Acta., 450, 73-5. (2006)

7. Blanco, L.H., Vargas, E.F., Suárez, A.: J. Therm. Anal. Calorim., 104, 100-104. (2011)

8. Clavijo-Penagos, J.A., Gracia-Fadrique, J.: Bol. Soc. Quím. Mex., 4, 84. (2010)

9. Clavijo, J.A., Blanco, L.H.: J. Solution Chem., DOI: 10.1007 / s10953-011-9736-9 (2011)

10. Clavijo Penagos, J.A., Blanco, L.H.: J. Chem. Thermodyn., DOI:10.1016 / j.jct.2011.08.024 (2011)

11. Clavijo-Penagos, J.A., Gracia-Fadrique, J.: Surface behavior of hexamethylenetetramine in aqueous solution. The $45^{\text {th }}$ Mexican Congress of Chemistry and $29^{\text {th }}$ National Congress of Chemical Education, Riviera Maya, Quintana Roo, Mexico (2010)

12. Clavijo, J., Blanco, L.: Temperature dependence of partial molal volume at infinite dilution for two macrocyclic aminals in aqueous solution between 275.15 and 278.15 K. In: $19^{\text {th }}$ European conference on thermophysical properties, Thessalonica, Greece, (2011) 
